\title{
Percepções e conhecimentos de pacientes com Doença Renal Crônica em tratamento conservador ${ }^{1}$
}

\section{Perceptions and knowledge of patients with Chronic Kidney Disease under conservative treatment}

\author{
Percepciones y conocimientos de pacientes con Enfermedad Renal Crónica en tratamiento \\ conservador
}

Tatiana Camila GricioI, Luciana KusumotaII , Marília de Lima Cândido ${ }^{I I I}$

\footnotetext{
${ }^{1}$ Pesquisa de Iniciação Científica, financiada pela Fundação de Amparo à Pesquisa do Estado de São Paulo.

${ }^{\text {I }}$ Enfermeira. Jaboticabal, SP. E-mail: tatiana.gricio@usp.br.

II Enfermeira. Doutora em Enfermagem. Docente do Departamento de Enfermagem Geral e Especializada da Escola de Enfermagem de Ribeirão Preto da Universidade de São Paulo (EERP/USP). Ribeirão Preto, SP. E-mail: kusumota@eerp.usp.br.

III Aluna de graduação em Enfermagem da EERP/USP. Ribeirão Preto, SP. E-mail: mariliadelimacandido@yahoo.com.br.
}

\section{RESUMO}

A Doença Renal Crônica (DRC) é uma síndrome irreversível e progressiva das funções glomerular, tubular e endócrina dos rins. Há evidências de que intervenções adequadas e precoces podem retardar sua progressão. Foi objetivo deste estudo identificar e caracterizar pacientes em tratamento conservador e identificar suas percepções e conhecimentos em relação à DRC e tratamentos. O referencial metodológico foi a história oral temática. A coleta de dados ocorreu de agosto a setembro de 2007. Dos 20 pacientes entrevistados no ambulatório de uremia do Hospital das Clínicas de Ribeirão Preto, 12 (60\%) eram homens e 8 (40\%) mulheres, com idade média de 58,6 anos. Dentre as comorbidades, destaca-se o elevado número de hipertensos (85\%) e diabéticos (45\%). Das entrevistas emergiram três categorias: 1) Conceitos imprecisos da DRC; 2) Tratamento atual; 3) Tratamento futuro. Conclusão: em sua maioria, pacientes em tratamento conservador possuem informações insuficientes a respeito da DRC e tratamentos, o que pode interferir na adesão e, consequentemente, acelerar a progressão da doença. Evidencia-se a necessidade de se construir uma abordagem educativa como estratégia para estimulá-los a aderirem ao tratamento conservador, diminuindo, assim, a morbidade e mortalidade no início e durante o tratamento dialítico.

Descritores: Insuficiência Renal Crônica; Enfermagem; Conhecimento.

\section{ABSTRACT}

Chronic Kidney Disease (CKD) is an irreversible and progressive syndrome of the glomerular, tubular and endocrine functions of the kidneys. There are evidences that adequate and early interventions can retard its progression. Objectives: to identify e characterize patients under conservative treatment and identify their perceptions and knowledge about CKD and treatments. The methodological framework used was the thematic oral history, in for data collection, which was carried out between August and September 2007. Among the 20 patients interviewed in the uremia outpatient clinic at Hospital das Clínicas in Ribeirão Preto, 12 (60\%) were men and 8 (40\%) women, aged 58,6 years in average. Among the comorbidities, the high number of patients with hypertension (85\%) and diabetes (45\%) is highlighted. From interviews emerging three categories: 1) Inaccurate concepts about CKD; 2) Current treatment; 3) Future treatment. Conclusion: most patients under conservative treatment have insufficient information about CKD and treatments, what can interfere in adhesion and, consequently, accelerate disease progression. The need of building an educative approach as strategy to stimulate patients to adhere to conservative therapy is shown, thus reducing morbidity and mortality in the beginning and during dialysis treatment.

Descriptors: Renal Insufficiency; Chronic; Nursing; Knowledge.

\section{RESUMEN}

La enfermedad renal crónica (ERC) es una síndrome irreversible y progresiva de las funciones glomerular, tubular y endócrina de los riñones. Hay evidencias de que intervenciones adecuadas y precoces pueden retardar su progresión. Objetivos: Identificar y caracterizar los pacientes en tratamiento conservador e identificar sus percepciones y conocimientos en relación a la ERC y tratamientos. El referencial metodológico fue la historia oral temática, la recolecta de datos realizada entre agosto y setembro 2007. Entre los 20 pacientes entrevistados en la clínica ambulatoria de uremia del Hospital das Clínicas de Ribeirão Preto, 12 (60\%) eran hombres y 8 (40\%) mujeres, con edad media de 58,6 años. Entre las comorbilidades, se destaca el alto número de hipertensos (85\%) y diabéticos (45\%). Las entrevistas emergiendo tres categorías: 1) Conceptos imprecisos de la ERC; 2) Tratamiento actual; 3) Tratamiento futuro. Conclusión: pacientes en tratamiento conservador tienen informaciones insuficientes acerca de la ERC y tratamientos, lo que puede interferir en la adhesión y, consecuentemente, acelerar la progresión de la enfermedad. Se evidencia la necesidad de se construir un enfoque educativo como estrategia para estimular los pacientes a adherir al tratamiento disminuyendo la morbilidad y mortalidad en el inicio y durante el diálisis.

Descriptores: Insuficiencia Renal Crónica; Enfermería; Conocimiento. 


\section{INTRODUÇÃO}

A Doença Renal Crônica (DRC) é conceituada como uma síndrome irreversível e progressiva das funções glomerular, tubular e endócrina dos rins, caracterizada por uma filtração glomerular menor que $60 \mathrm{ml} / \mathrm{min} / 1,73 \mathrm{~m}^{2}$ durante um período de três meses ou mais ${ }^{(1)}$. Os rins tornam-se, portanto, incapazes de manter o equilíbrio metabólico e hidroeletrolítico, o que resulta em uremia(2-3).

As fases de redução das funções renais são: estágio 0 , com risco aumentado, Taxa de Filtração Glomerular (TFG) maior ou igual a $90 \mathrm{ml} / \mathrm{min} / 1,73 \mathrm{~m}^{2}$ e presença de fatores de risco para doença renal crônica; estágio 1, com lesão renal e TFG normal ou aumentada (maior ou igual $90 \mathrm{ml} / \mathrm{min} / 1,73 \mathrm{~m}^{2}$ ); estágio 2, com lesão renal (discreta) e ligeira diminuição da TFG $\left(60-89 \mathrm{ml} / \mathrm{min} / 1,73 \mathrm{~m}^{2}\right)$; estágio 3, com diminuição moderada da TFG (30-59 $\left.\mathrm{ml} / \mathrm{min} / 1,73 \mathrm{~m}^{2}\right)$; estágio 4 , com diminuição grave da TFG $\quad\left(15-29 \mathrm{ml} / \mathrm{min} / 1,73 \mathrm{~m}^{2}\right) ; \quad$ estágio 5 é caracterizado pela Doença Renal Crônica (DRC) terminal com TFG menor que $15 \mathrm{ml} / \mathrm{min} / 1,73 \mathrm{~m}^{2(1)}$.

A diminuição progressiva da função renal leva fatalmente à uremia. Na maioria dos pacientes, a sintomatologia urêmica torna-se evidente quando a TFG atinge $15-20 \mathrm{ml} / \mathrm{min} / 1,73 \mathrm{~m}^{2(2)}$. Os principais sinais e sintomas descritos são: fraqueza, fadiga, confusão mental, cefaleia, prurido, edema, hálito de amônia ("hálito urêmico"), náusea, vômito, anorexia, constipação, diarreia, anemia, infertilidade, cãibras musculares, osteodistrofia renal, entre outros. A intensidade destes na DRC depende do grau de comprometimento renal e de outras condições subjacentes, bem como a idade da pessoa(3). Nas fases iniciais, ocorre adaptação do organismo e o paciente apresenta-se assintomático, porém nas fases mais tardias os sintomas são intensos e persistentes. Com o início da Terapia Renal Substitutiva (TRS), os sinais e sintomas mais intensos tendem a diminuir ou desaparecer(2).

Dentre as causas de DRC estão as doenças renais primárias (glomerulonefrites, pielonefrites e doenças obstrutivas), doenças sistêmicas (hipertensão arterial grave, diabetes mellitus, doenças auto-imunes, gota complicada, amiloidose e mieloma múltiplo), doenças hereditárias (rins policísticos, síndrome de Alport e cistinose) e malformações congênitas (agenesia renal, hipoplasia renal bilateral e válvula de uretra posterior) ${ }^{(4)}$.

Inquéritos epidemiológicos têm apontado como principais causas da Doença Renal Crônica (DRC) terminal a nefro esclerose $(27,1 \%)$, e diabetes mellitus $(22,3)^{(5)}$. Ressalta-se a importância da hipertensão arterial e da diabetes mellitus como doenças da modernidade que acometem principalmente adultos de meia idade, os quais podem viver durante longo período sem manifestação de sintomatologia, embora haja comprometimento, dentre outros órgãos, dos rins. Essas doenças também podem evoluir para o prejuízo das funções renais devido ao seguimento inadequado dos seus respectivos tratamentos. São, portanto, doenças que preocupam os profissionais da saúde em Nefrologia no âmbito da prevenção e controle da DRC.

O tratamento de DRC depende da evolução da doença, podendo ser conservador com uso de medicamentos, dietético e restrição hídrica; quando esse tratamento torna-se insuficiente, é necessário iniciar a diálise que substitui, em parte, a função dos rins, ou ainda candidatar-se a um transplante renal(2).

O tratamento conservador tem como meta auxiliar a redução do ritmo da progressão da doença renal, utilizando-se de orientações dietéticas que objetivam a promoção de um estado nutricional adequado, controle dos distúrbios metabólicos e da sintomatologia urêmica( ${ }^{(6)}$. $\mathrm{O}$ tratamento medicamentoso objetiva o controle das doenças crônicas instaladas, bem como a correção de distúrbios metabólicos e urêmicos. A restrição hídrica pode ser necessária para aqueles pacientes que durante as fases de redução na TFG apresentam diminuição do volume de diurese produzido.

A DRC é uma doença de alta morbidade e mortalidade, com aumento progressivo da incidência e prevalência nas populações mundiais. Estas taxas variam a depender do grau de desenvolvimento das regiões geográficas, pois as condições socioeconômicas, demográficas e ambientais influenciam na determinação dessas variáveis. A incidência de novos pacientes em tratamento dialítico cresce cerca de $8 \%$ ao ano(5). Contudo, não há publicações nacionais referentes a dados epidemiológicos precisos de pacientes com DRC em período pré-dialítico, o que evidencia uma subnotificação dos dados reais e de óbitos, principalmente nas regiões menos desenvolvidas.

Segundo dados publicados na literatura, a melhoria das condições de saúde poderia contribuir para a obtenção de melhores resultados relacionados à diálise e aos pacientes com DRC nas diferentes fases. A partir disto, tornou-se evidente que intervenções adequadas e precoces, que englobem todo o espectro da DRC, podem retardar a progressão da doença, evitando a perda da função renal, ou ainda melhorar a disfunção orgânica e condições co-mórbidas nos pacientes que progridem para a DRC terminal(1).

A realização do diagnóstico da DRC precocemente, ou seja, quando os rins ainda preservam parcialmente suas funções, e a realização do tratamento conservador podem contribuir para uma melhor condição clínica, psicológica e social do paciente, ao iniciar e manter-se em tratamento por diálise $^{(7)}$. 
Neste contexto, pesquisas sobre dados epidemiológicos e de opiniões pessoais de pacientes com DRC em tratamento conservador representam uma contribuição valiosa para a assistência de enfermagem, pois os resultados obtidos podem contribuir para a adoção de estratégias que priorizem a adesão dos pacientes ao tratamento e a elaboração de orientações sobre a DRC, tratamento conservador, diálise e transplante renal.

Este estudo teve como objetivo identificar os pacientes em tratamento conservador da DRC atendidos em um hospital público no município de Ribeirão Preto-SP; caracterizá-los quanto aos dados socioeconômicos, demográficos e clínicos e identificar as suas percepções e conhecimentos em relação ao processo saúde-doença e tratamentos.

\section{TRAJETÓRIA METODOLÓGICA}

Para o desenvolvimento deste estudo foi utilizada a "história oral" devido à adequação dos princípios da abordagem qualitativa que designam este método.

História oral é um método que vem sendo usado para captar experiências de pessoas dispostas a falar sobre aspectos de sua vida, sem afastar-se do contexto social. É utilizado para elaboração de documentos, arquivamentos e estudos referentes à vida social das pessoas ${ }^{(8)}$.

Para atender à proposta deste trabalho acerca dos pacientes com DRC, em tratamento conservador, foi utilizada a modalidade da história oral temática, que busca identificar a opinião do entrevistado a respeito de um assunto específico, um tema préestabelecido; aborda questões diretivas, objetivas, factuais e temáticas e a condução do trabalho pelo entrevistado confere a esta modalidade características peculiares ${ }^{(8)}$.

A pesquisa foi realizada com pacientes em tratamento conservador da DRC no ambulatório de uremia do Hospital das Clínicas da Faculdade de Medicina de Ribeirão Preto - Universidade de São Paulo que atenderam aos seguintes critérios de inclusão: apresentar 18 anos ou mais de idade; ter diagnóstico de DRC em tratamento conservador da função renal; aceitar participar do estudo e assinar o termo de consentimento livre e esclarecido.

Para a coleta de dados, que ocorreu de agosto a setembro de 2007, foi realizada uma entrevista e aplicado um instrumento de caracterização dos pacientes do ponto de vista socioeconômico, demográfico e clínico, adaptado para este estudo, contendo: idade, sexo, cor da pele, estado civil, número de filhos vivos, escolaridade, renda, atividade atual, profissão exercida por mais tempo, com quem vive, tempo de diagnóstico da DRC, tempo de realização do tratamento conservador, complicações físicas associadas à DRC, causa etiológica da DRC, co-morbidades, uso de medicamentos, apoio informal, acompanhamento psicológico e nutricional e uso de eritropoetina. As complicações físicas associadas à DRC consideradas foram: cãibras, hipotensão e/ou hipertensão arterial, cefaleia, fraqueza, dor, infecções recorrentes, infertilidade, anemia, prurido, perda ou ganho de peso, constipação intestinal, arritmia cardíaca e outras.

Para identificar as percepções e conhecimentos do paciente a respeito de sua doença, tratamento atual e possibilidade de tratamentos futuros foi realizada a gravação das falas em resposta às questões norteadoras: "O que é Doença Renal Crônica para o (a) senhor(a)? Como o(a) senhor(a) percebe o seu tratamento atual e as possibilidades de tratamentos futuros?". As entrevistas duraram, em média, 30 minutos.

Foi solicitada a permissão para realização da pesquisa ao responsável pelo atendimento no ambulatório de uremia do HCFMRP-USP, onde aconteceu a coleta de dados. O projeto acatou as normas da Resolução CNS196/96, tendo sido aprovado pelo Comitê de Ética do Hospital das Clínicas da Faculdade de Medicina de Ribeirão Preto Universidade de São Paulo (Processo n.7799/2006).

Para cada paciente, foi solicitada sua participação no estudo utilizando-se de um termo de Consentimento Livre e Esclarecido, contendo em linguagem clara e simples informações referentes à pesquisa, inclusive que as entrevistas seriam gravadas e que seria assegurado o anonimato dos participantes. Todos os pacientes concordaram em participar da pesquisa e assinaram o referido termo.

Os dados coletados foram organizados de forma a facilitar a digitação, a conferência e a análise dos mesmos. Elaborou-se uma planilha no programa Excel for Windows, no qual os dados foram digitados duplamente, validados e conferidos, a fim de eliminar os erros.

Na sequência, foi realizada a caracterização dos sujeitos estudados por meio dos instrumentos referentes às questões socioeconômicas, demográficas e clínicas.

As respostas às questões norteadoras foram gravadas em fita de áudio e a seguir foram submetidas às seguintes etapas de análise: transcrição, textualização e transcriação(8).

Após a identificação dos conteúdos expressos nas entrevistas e anotação dos conceitos recorrentes, foi possível realizar a categorização das histórias obtidas com os pacientes e a partir disto analisálas ${ }^{(8)}$.

\section{RESULTADOS E DISCUSSÃo}

Participaram do estudo 20 pacientes em tratamento conservador da DRC um hospital público em Ribeirão Preto, estado de São Paulo. 
Conforme as variáveis investigadas, a idade variou de 21 a 74 anos, com uma média de 58,6 anos. Quanto ao sexo, 12 (60\%) eram homens e 8 $(40 \%)$ eram mulheres. Observou-se, a aproximação com os resultados de estudo, os quais mostraram que em 1.076 casos analisados entre 1992 e 1994, a DRC era mais frequente em homens $(60 \%)^{(9)}$. Em relação à cor da pele, $10(50 \%)$ eram brancos, 6 $(30 \%)$ negros e $4(20 \%)$ pardos. A DRC tem acometido predominantemente brancos ${ }^{(9-10)}$.

Quanto ao estado conjugal, 12 (60\%) moravam com esposo(a) ou companheiro(a); 6 (30\%) nunca se casaram ou moraram com companheiro(a); 1 $(5 \%)$ era separado(a) e 1 (5\%) era viúvo(a). o número de filhos vivos variou de 0 a 15 filhos, com uma média de 3,5 filhos. Foi verificado que 14 (70\%) pacientes moravam com a família, $4(20 \%)$ apenas com o esposo(a) e 2 (10\%) moravam na casa de outra pessoa.

$\mathrm{O}$ fato dos pacientes terem e/ou conviverem com familiares pode ser um aspecto positivo, pois a DRC pode progredir com complicações físicas, sociais e emocionais e/ou incapacidades, demandando cuidado informal e formal. $O$ apoio de pessoas significativas pode ser de extrema valia, pois os pacientes em estágios avançados da DRC (4 e 5) tendem a apresentar sobrecarga similar aos pacientes em diálise e pacientes com câncer ${ }^{(11)}$.

Referente à escolaridade, $18(90 \%)$ sabiam ler e escrever e $2(10 \%)$ eram analfabetos; encontrou-se a média de quatro anos de estudo, o que confirma os achados da realidade nacional brasileira. Esta característica é de extrema importância no que tange a temática educação em saúde, que tem sido explorada na abordagem do paciente renal crônico em tratamento conservador ${ }^{(10,12-14)}$, pois o grau de escolaridade influencia diretamente no processo ensino-aprendizagem entre profissional da saúde e paciente. Deve-se destacar a necessidade de se ampliar os estudos desta temática na realidade brasileira, visto que os investimentos dos profissionais da saúde na assistência à prevenção e progressão da DRC são recentes, de meados da década de noventa, e consequentemente, poucas pesquisas têm sido realizadas.

Abordando as condições de saúde-doença, os pacientes haviam recebido o diagnóstico de DRC, em média, há 6,1 anos, variando de 2 a 252 meses; já o tempo de duração do tratamento conservador foi, em média, de 5,3 anos, o que revela que alguns pacientes não iniciaram o tratamento imediatamente após receberem o diagnóstico da DRC. Quanto às complicações associadas à doença, 17 (85\%) referiram hipertensão arterial, 13 (65\%) cãibras, 13 (65\%) referiram dor em outra região do corpo, 12 (60\%) fraqueza, $12(60 \%)$ perda de peso, $9(45 \%)$ anemia, 6 (30\%) dores de cabeça, 5 (25\%) relataram apresentar outros tipos de complicações, 4
(20\%) prurido, 4 (20\%) ganho de peso, $4(20 \%)$ constipação intestinal, 4 (20\%) arritmia, 2 (10\%) infecções repetitivas e $1(5 \%)$ referiu infertilidade.

Segundo os pacientes, a causa da DRC foi: 6 (30\%) diabetes mellitus, 6 (30\%) não especificaram a causa, $4(20 \%)$ hipertensão arterial, $3(15 \%)$ referiam outra causa e $1(5 \%)$ rins policísticos. A não realização do diagnóstico da doença renal na ocasião de ocorrência da doença de base repercute em dificuldades para confirmar a causa real da falência renal, sejam elas investigadas nos registros de prontuários médicos ou questionadas aos pacientes. É de extrema relevância reconhecer o quadro das doenças que causam a DRC, pois isto pode contribuir para o planejamento da assistência de enfermagem no que tange a prevenção e controle da progressão da DRC junto à população geral.

Quanto às comorbidades, $17(85 \%)$ referiram hipertensão arterial, 15 (75\%) déficit visual, 9 (45\%) diabetes mellitus, $8(40 \%)$ referiram outro tipo de comorbidade; $4(20 \%)$ tinham varizes, $4(20 \%)$ catarata, $3(15 \%)$ doença cérebro-vascular, 3 (15\%) déficit auditivo, $2(10 \%)$ apresentavam insuficiência cardíaca, $2(10 \%)$ haviam sofrido infarto do miocárdio; 2 (10\%) neoplasia maligna, 2 (10\%) osteopatias e $1(5 \%)$ neoplasia benigna. Destaca-se - elevado número de pacientes com hipertensão arterial e diabetes mellitus, que são consideradas, atualmente, as principais causas da DRC e importantes fatores que contribuem na progressão desta doença $(5,15)$.

Somente $4(20 \%)$ dos pacientes recebiam ajuda para realizar atividades do dia-a-dia; 4 (20\%) tiveram acompanhamento nutricional e $1(5 \%)$ teve acompanhamento psicológico, sendo que não necessariamente tais acompanhamentos eram realizados pelo serviço referido. Tais aspectos são relevantes no tratamento do paciente renal crônico do ponto de vista multidisciplinar. Contudo, o serviço investigado ainda não possui uma equipe de profissionais da saúde disponível para atender estes pacientes.

Em relação aos resultados sobre o conhecimento e percepções dos pacientes sobre a DRC foi encontrado uma diversidade de atribuições de conceitos e adjetivos. Dois dos pacientes não souberam definir a DRC, os demais atribuíram conceitos breves, relacionados à falência dos rins ou expressões negativas, tais como: a DRC gera limitações e dificuldades; traz conseqüências ruins; traz mudanças na vida; contribui para a piora da saúde.

Em relação aos resultados sobre o conhecimento e percepções sobre o tratamento conservador, foi referido consistir a abordagem da dieta (17 pacientes); uso de medicamentos (16 pacientes); repouso e exercícios físicos ( 2 pacientes). 
Quanto aos resultados sobre o conhecimento e percepções sobre os tratamentos futuros observou-se que os pacientes mencionaram saber da existência da hemodiálise e diálise peritoneal, contudo foram relatos breves e incertos sobre cada modalidade dialítica.

A trajetória histórica foi construída a partir das entrevistas e questões norteadoras emergindo três categorias: 1 - Conceitos imprecisos da DRC, 2 Tratamento atual e 3 - Tratamentos futuros, que serão descritas e podem ser observadas na Figura 1: 


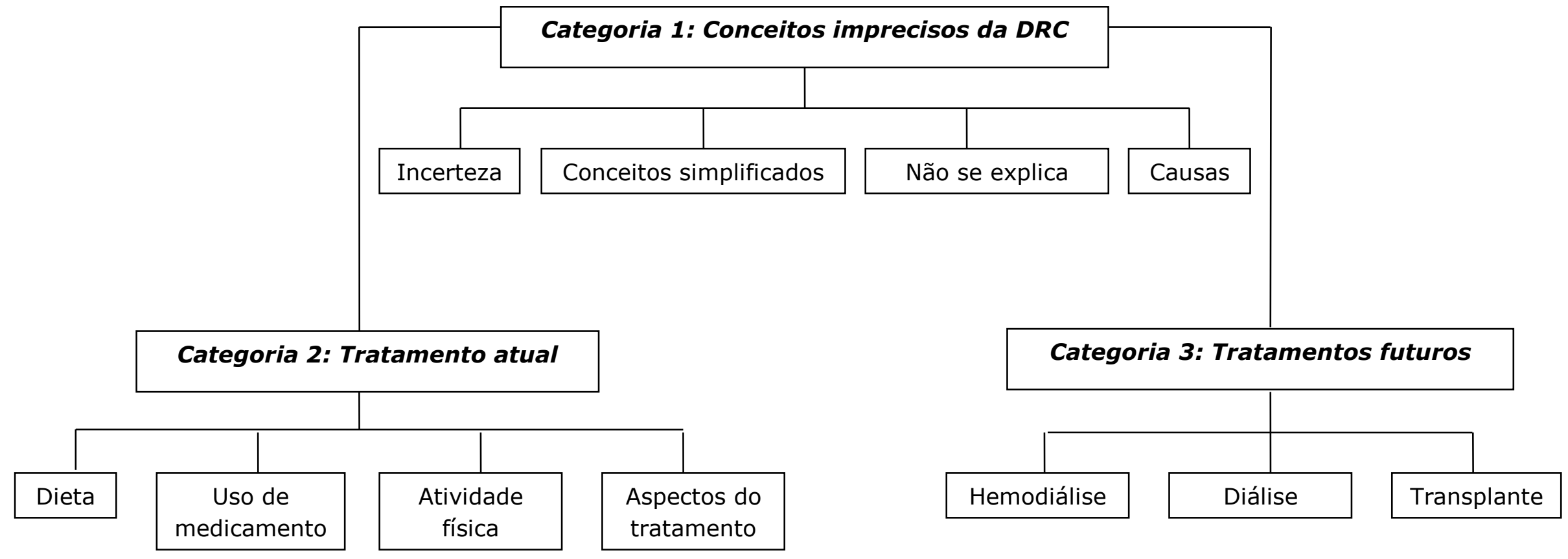

Figura 1: Categorias 1, 2 e 3 e suas respectivas subcategorias que emergiram da análise das falas dos pacientes com DRC em tratamento conservador da função renal. Ribeirão Preto, 2007 
De maneira geral, os pacientes demonstraram pouco conhecimento a respeito da doença que possuem, do tratamento que estão realizando e também das modalidades de tratamentos que existem, apesar da equipe médica e enfermeira, responsáveis pelo atendimento destes pacientes, realizarem orientações verbais durante as consultas. Dentre os objetivos do atendimento neste ambulatório está o de preparar os pacientes para o início do tratamento dialítico, visto que se apresentam, segundo o National Kidney Foundation $^{(1)}$, na fase de redução das funções renais de estágio 4, com diminuição grave da TFG (15-29 $\mathrm{ml} / \mathrm{min} / 1,73 \mathrm{~m}^{2}$ ). O preparo para o início da diálise compõe um dos aspectos do tratamento conservador, sendo, portanto, imprescindível que os pacientes demonstrem conhecimentos a respeito da sua doença, tratamento conservador e da diálise, até para fazer a opção pela modalidade de tratamento a realizar(12-14).

Em relação à categoria 1 "Conceitos imprecisos da DRC", foram revelados conceitos simplificados, incertezas, relação com as causas e ainda a falta de explicação para as suas condições de saúde atuais, que podem ser exemplificadas por meio das falas abaixo:

É difícil dizer o que é a Doença Renal Crônica. Eu sei que não é boa coisa, é ruim... (2)

Eu não sei direito o que é a Doença Renal Crônica. Acho que é uma complicação da diabetes, da pressão, mas não sei dizer o que causa no corpo... (4)

O que eu sei sobre a Doença Renal Crônica é o que falaram para mim, que é causada pelo diabete, pois eu tenho esta doença desde os treze anos de idade, e hoje estou com cinquenta e dois. Então é esse diabete antigo que está causando esse problema no rim, e que vai piorar cada vez mais... (8)

A Doença Renal Crônica é quando o rim não está funcionando, não está filtrando direito... (12)

Apesar de simplificados os conceitos mencionados pelos pacientes a respeito da DRC, foram observados poucos aspectos equivocados. Os significados da doença revelaram-se de diferentes formas para os pacientes investigados provavelmente devido às percepções individuais de manifestação da DRC. O quadro clínico de aparecimento dos sinais e sintomas decorrentes de complicações de outras doenças que levaram à falência renal frequentemente é tardio, o que leva os pacientes a postergarem a procura de atendimento em saúde, retardando o início do tratamento(2).

Em relação às causas mencionadas, destacaramse a diabetes mellitus e hipertensão arterial, que são considerados, na atualidade, importantes fatores que contribuem na progressão da $\operatorname{DRC}^{(5,15-16)}$.

A diabetes mellitus e a hipertensão quando não controladas causam, de formas diferentes, lesões progressivas e lentas em órgãos alvos, dentre eles os rins que, com o passar do tempo, desenvolvem a perda irreversível da função parcial e/ou total(16).

Atrelado ao conceito de DRC que os pacientes referiram, apareceram os significados do tratamento conservador da função renal, que foi chamado, para fins de análise, de tratamento atual. Os pacientes se referiram a este tratamento mencionando a realização de dieta, uso de medicamentos, realização de atividade física e ainda outros aspectos relevantes relacionados também com o preparo para a diálise:

Eles orientam repouso, fazer dieta, tomar o remédio na hora certa, não abusar na comida, não abusar no serviço, fazer o que eu agüentar. Acho que está dando resultado porque se eu não tivesse fazendo o tratamento eu já tinha morrido há muito tempo.... Agora, os médicos me deram um papel para dilatar uma veia, que é para fazer hemodiálise... (2)

O remédio também faz parte do tratamento, porque se não tomar... Antes eu tomava quinze comprimidos, agora eu tomo quatro e percebo que estou sentindo a mesma coisa. Eu me sinto bem... (3)

O tratamento que eu estou fazendo aqui está bom, eu tomo remédio e também controlo a comida. Tem muita coisa que eu não como: carne de porco, carne gorda, às vezes como só um pedacinho. Eu gostava muito de churrasco, mas hoje em dia não frequento mais. Bebida alcoólica também não bebo, nem refrigerante porque eu tenho medo... (5)

Minha esposa me ajuda a fazer o tratamento, em casa ela faz a comida com pouco sal, sem pimenta, para não me prejudicar; a alimentação é muito importante para o tratamento. Tem até um ditado que diz que o peixe morre pela boca; se pela minha boca entra medicamentos, eu não vou comer algo que eu sei que me faz mal, então nós temos que controlar nós mesmos... (7)

... Fiquei um bom tempo sem tratamento, não fazia dieta, comia normal. Quando vim para cá, minha creatinina já estava bem alta... até fístula eu já fiz. Mas estou me cuidando, consumo pouquíssimo sal, não como feijão, milho, ervilha, faço isso pra evitar o ácido úrico, pois o médico disse que era isso que estava prejudicando meu rim. Além da alimentação, eu faço exercícios, tomo remédio para hipertensão e insulina para o diabetes... (10)

O tratamento conservador inclui a monitorização e terapêutica das doenças causadoras da DRC, bem como a determinação de restrições dietéticas, controle da pressão arterial, da acidose metabólica, da hipercalemia, da anemia e da osteodistrofia renal(16).

A avaliação e o manejo nutricional dos pacientes em tratamento conservador podem assegurar o retardamento da progressão da doença, a redução da toxicidade urêmica e a manutenção de um adequado 
estado nutricional, prevenindo, assim, a desnutrição( 2,6$)$.

O estabelecimento de uma dieta hipoprotéica é baseado nos seguintes critérios: grau da DRC, presença de progressão desta, grau de proteinúria e uso ou não de glicocorticóides ${ }^{(2)}$. Até o estágio 2 não é indicada a redução da ingestão protéica, exceto se houver sinais de progressão da DRC. O objetivo nesta fase é o controle de outros fatores, tais como pressão arterial e hiperlipidemia. A partir do estágio 3 é recomendado iniciar uma dieta com 0,6-0,8 g/ $\mathrm{kg} /$ dia de proteínas, sendo $60 \%$ de alto valor biológico (proteínas animais) e controle rigoroso da pressão arterial. Para assegurar um balanço nitrogenado neutro ou positivo, a restrição protéica deve ser acompanhada de adequada ingestão calórica (35 $\mathrm{Kcal} / \mathrm{kg} / \mathrm{dia}$ ). É importante ressaltar que pacientes em estágio 5 estão mais suscetíveis à desnutrição e, portanto, devem ser monitorizados quanto aos sinais precoces desta, como a perda de peso e diminuição da concentração plasmática de creatinina $(2,10,16)$.

É recomendada ainda uma dieta com aproximadamente $2 / 3$ de carboidratos e $1 / 3$ de lipídios, sendo estes ricos em mono- e poliinsaturados, cujo uso é imprescindível para minimizar os riscos de hiperlipidemia e, consequentemente, para amenizar as doenças cardiovasculares frequentemente associadas à $\mathrm{DRC}^{(2)}$. A restrição hídrica na dieta não é necessária a menos que haja uma redução significativa da função renal ( $<5 \mathrm{ml} / \mathrm{min}$ ). O controle hídrico pode ser feito através da monitoração cuidadosa do peso corporal e do volume de excreção urinária(2).

Em relação ao controle da pressão arterial, é realizado por meio de um tratamento antihipertensivo baseado na redução da ingestão de sódio, sendo recomendado ingestão máxima de 3g/dia, e na utilização de medicamentos, sendo frequentemente necessário associar dois ou mais anti-hipertensivos para controle adequado ${ }^{(2,16)}$.

Uma das complicações graves da DRC é a osteodistrofia renal (OR), que engloba um conjunto de desordens esqueléticas consequentes às alterações do metabolismo do cálcio e do fósforo. Sua prevenção e tratamento consistem em na avaliação e intervenções em relação às concentrações séricas de cálcio e fósforo funcionamento das paratireóides, integridade óssea e calcificação de tecidos moles(17).

Outra complicação da DRC é a anemia, a qual é frequentemente encontrada em pacientes no estágio 4 da redução das funções renais, sendo comumente causada pela deficiência de eritropoetina. Além desta, estados inflamatórios, perdas sangüíneas, deficiências de ferro, de ácido fólico e de vitamina B12 podem contribuir para a ocorrência da anemia e devem ser investigadas antes da introdução de terapia de reposição de eritropoetina( $(2,18)$. Pacientes que apresentam valores de ferritina $<100 \mathrm{ng} / \mathrm{ml}$ e saturação da transferrina menor que $20 \%$ necessitam de reposição rápida de ferro por via endovenosa. O tratamento com eritropoetina é indicado quando a hemoglobina atingir valor igual ou inferior a $11 \mathrm{~g} / \mathrm{dl}$ e deverá ser complementado com ácido fólico 2 a 5 $\mathrm{mg} / \mathrm{dia}^{(2,16,18)}$.

As mudanças no estilo de vida são aconselhadas principalmente por estarem associadas à redução do risco de eventos cardiovasculares, controle da pressão arterial e da diabetes. Dessa maneira, tornase necessário a redução de peso, da ingestão de álcool, realização de atividades físicas regulares e interrupção do hábito de fumar ${ }^{(2,16)}$.

Diante dos resultados encontrados neste estudo, o acompanhamento psicológico dos pacientes não faz parte da assistência prestada no serviço, pois apenas um mencionou ter recebido este tipo de atendimento. A fase pré-dialítica exige dos pacientes grandes capacidades de adaptação às novas situações que, quando não são desenvolvidas, podem levar ao aumento da prevalência de transtornos mentais. Desta maneira, torna-se necessário um acompanhamento profissional adequado, que se constitua como um elemento decisivo para o sucesso do tratamento do paciente renal crônico(19).

Recomenda-se, também, que os pacientes procurem atividades prazerosas e relaxantes e que não trabalhem excessivamente, mesmo não se tendo evidências dos benefícios do controle do estresse para eles(2).

A terceira categoria, chamada de Tratamentos futuros, evidenciou as considerações dos pacientes a respeito das possibilidades de tratamentos que eles poderão realizar com a progressão da DRC para a fase terminal da doença. Percebeu-se que os pacientes possuíam poucas informações das modalidades de diálise e transplante renal, às vezes nenhuma:

Em relação aos tratamentos futuros eu não sei nada... (4)

Me disseram que os meus rins estão funcionando só $13 \%$ cada um, então vai precisar fazer a fístula para fazer a hemodiálise quando precisar... Já ouvi dizer também que tem um outro tipo de tratamento, que implanta um aparelho na barriga, que faz em casa. Mas para mim é difícil, minha visão é ruim, minha família já é de idade, não tem quem faça, e também tem o perigo da infecção, por isso eu acho melhor fazer a hemodiálise no hospital. (8)

Em relação aos tratamentos futuros, eu conheço a diálise, que é a troca de bolsa, e a hemodiálise. Minha mãe também teve Insuficiência Renal Crônica, ela fez diálise até morrer. Eu acompanhava o tratamento dela... (9)

Além do tratamento conservador, conheço também a hemodiálise e a diálise peritoneal. Eu os conheço porque eu tive uma irmã que morreu de Insuficiência Renal... inclusive iria fazer transplante, mas não 
aceitou; ela morreu porque não aceitava, mas nós temos que aceitar as coisas, não tem jeito. (10)

Não fui eu quem escolhi fazer hemodiálise, mas já me falaram sobre ela, me explicaram e até já me mostraram. Além desse, não conheço outro tipo de tratamento. (11)

... eu conheço só a hemodiálise, é um tratamento muito difícil e eu espero que Deus me abençoe e que eu não precise fazer mais. Eu já precisei fazer duas vezes e não foi fácil.... (15)

Como tratamentos dialíticos existem: Hemodiálise e Diálise Peritonial (Diálise Peritonial Ambulatorial Contínua - DPAC, Diálise Peritonial Intermitente- DPI, Diálise Peritonial AutomatizadaDPA).

A diálise é um processo físico-químico que se utiliza de mecanismos de transporte de solutos (difusão), solvente (ultrafiltração) e convecção por meio de uma membrana semipermeável, podendo ocorrer mediante o uso de hemodiálise ou diálise

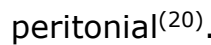

A hemodiálise é o tratamento dialítico mais utilizado na atualidade. Consiste na diálise promovida por uma máquina, em que a filtração do sangue é realizada fora do organismo; a média de sessões semanais é de três, por um período de três a cinco horas por sessão, dependendo das necessidades individuais ${ }^{(20)}$.

Diálise peritonial é outro tipo de diálise realizada por meio do peritônio, o qual atua como membrana semipermeável entre o organismo e uma determinada quantidade de líquido para diálise, composto por solução de glicose e eletrólitos, capaz de retirar do sangue o excesso de líquido, substâncias endógenas e exógenas e tóxicas ao organismo, restabelecendo o equilíbrio hidroeletrolítico e ácido-básico. Nesta modalidade, estão a DPAC, DPI e DPA. Os pacientes candidatos a realizar a Diálise Peritonial precisam passar por um processo de ensino-aprendizagem, frequentemente desenvolvido pelo enfermeiro, para desenvolver a habilidade para realizar o procedimento técnico do tratamento em domicílio(20).

Transplante renal é uma terceira opção de tratamento para a DRC; envolve o transplante de um rim saudável de doador vivo ou falecido humano para um receptor com DRC, que se mantém em tratamento de diálise(20).

Os pacientes podem iniciar o tratamento por meio da hemodiálise ou diálise peritonial como primeira escolha ou após terem realizado as outras modalidades, a depender das condições e indicações individuais pela equipe profissional e a preferência do paciente e família. O transplante renal de doador vivo é indicado como primeira opção de tratamento em duas circunstâncias: para crianças e pacientes diabéticos com outras complicações físicas além da $\mathrm{DRC}^{(20)}$.
Estudos revelam que programas educativos prédiálise aumentam o conhecimento dos pacientes sobre a DRC, habilitando-os a decidirem a respeito das modalidades de tratamento futuro e contribuindo para maior bem-estar emocional e funcional, prevenção de complicações, podendo retardar a progressão da $\operatorname{DRC}^{(10,12)}$. O conhecimento é uma condição que promove a mudança de comportamento, e no caso do doente renal em estágios iniciais da doença, é imprescindível a adesão ao tratamento para intervir na progressão da DRC(14)

\section{CONCLUSÃO}

A temática do paciente renal crônico em tratamento conservador ainda é pouco explorada em nível nacional. Este fato trouxe dificuldades para contextualizar os resultados deste estudo com o quadro brasileiro, o que reverte em uma análise predominantemente de natureza descritiva dos achados. Recomenda-se o desenvolvimento de estudos com pacientes em tratamento conservador, em especial, relacionados aos dados epidemiológicos, educação e assistência de enfermagem, visando à otimização de atendimento a esses pacientes.

Em sua maioria, os pacientes com DRC em tratamento conservador possuem informações insuficientes a respeito da doença e tratamentos, o que pode interferir negativamente na adesão ao tratamento conservador e, consequentemente, acelerar a progressão da doença.

Evidencia-se, então, a necessidade de se construir uma abordagem educativa como estratégia para estimular os pacientes a aderirem ao tratamento, diminuindo a morbidade e mortalidade durante o tratamento conservador, assim como no início e durante o tratamento dialítico.

\section{REFERÊNCIAS}

1. National Kidney Foundation. K/DOQI clinical practice guidelines for chronic kidney disease: evaluation, classification, and stratification. Am J Kidney Dis. 2002 Feb;39(2 Suppl 1):S1-266.

2. Riella MC. Princípios de nefrologia e distúrbios hidroeletrolíticos. Rio de Janeiro: Guanabara Koogan; 2003.

3. Smeltzer CS, Bare BG, Hinkle JL, Cheever KH. Brunner \& Suddarth: Tratado de Enfermagem Médico-Cirúrgica. Rio de Janeiro: Guanabara Koogan, 2008.

4. Draibe AS, Ajzen H. Insuficiência renal crônica. In: Ajzen H, Schor N. Guias de Medicina Ambulatorial e Hospitalar - UNIFESP/Escola Paulista de Medicina: Nefrologia. Barueri: Manole; 2005. p. 183-96.

5. Romão Jr JE. Doença renal crônica: Definição, epidemiologia e classificação. J Bras Nefrol. 2004;26:1-3.

6. Nerbass FB, Feiten SF, Cuppari L. Nutrição do paciente com doença renal crônica em tratamento 
conservador. In: Barros E, Gonçalves LF. Nefrologia no consultório. Porto Alegre: Artmed; 2007. p. 42533.

7. Carvalho FJW, Machado AMEP. Idosos em consulta nefrológica inicial. Rev bras clín ter. $2001 ; 27(3): 101-$ 4.

8. Meihy JCSB, Holanda F. História oral: como fazer, como pensar. São Paulo: Contexto; 2007. p. 133-68.

9. Drey N, Roderick P, Mullee M. A population-based study of the incidence and outcomes of diagnosed chronic renal disease. Am J Kidney Dis. 2003;42(4):677-84.

10. Pacheco GS, Santos I, Bregman R. Características de clientes com DRC: evidências para o ensino do auto cuidado. Rev. enferm. UERJ. 2006;14(3):435-9. 11. Murphy EL, Murtagh FEM, Carey I, Sheerin NS. Understanding Symptoms in Patients with Advanced Chronic Kidney Managed Without Dialysis: Use of a short patient-completed assessment toll. Nephron Clin Pract. 2009;111(1):c74-80.

12. Klang $B$, Björvell $H$, Clyne N. Predialysis education helps patients choose dialysis modality and increases disease-specific knowledge. J Adv Nurs. $1999 ; 29(4): 869-76$.

13. Yeoh HH, Tiquia HS, Abcar AC, Rasgon SA, Idroos $\mathrm{ML}$, Daneshvari SF. Impact of predialysis care on clinical outcomes. Hemodial Int. 2003;7(4):338-41.

14. Mason J, Khunti K, Stone M, Farooqi A, Carr S. Educational Interventions in Kidney Disease Care: $A$ systematic review of randomized trials. Am J of Kidney Dis. 2008;51(6):933-51.

15. Mezzano $S$, Amair $P$, Wagner $P$. Guías para el cuidado del paciente con insuficiencia renal crônica em etapa de prediálisis. Buenos Aires: Sociedad Latinoamericana de Nefrología e Hipertensión/Anemia Working Group; 2004.

16. Draibe AS, Cendoroglo M. Tratamento conservador da insuficiência renal crônica. Revista Diagnóstico \& Tratamento. 2001;6(2):17-23.

17. Carvalho $A B$ de. Osteodistrofia Renal. J Bras Nefrol. 2004;26(3):29-39.

18. Abensur H. Anemia da Doença Renal Crônica. J Bras Nefrol. 2004;26(3):26-8.

19. Abdel-Kader $\mathrm{K}$, Unruh ML, Weisbord SD. Symptom burden, depression, and quality of life in chronic and end-stage kidney disease. Clin J Am Soc Nephrol. 2009;4(6):1057-64.

20. Canziani MEF, Draibe AS, Nadaletto MAJ. Técnicas dialíticas na Insuficiência Renal crônica. In: Ajzen $\mathrm{H}$, Schor N. Guias de medicina ambulatorial e hospitalar - UNIFESP/Escola Paulista de Medicina: Nefrologia. Barueri: Manole; 2005. p. 223-37.

Artigo recebido em 17.10.08.

Aprovado para publicação em 14.09.09.

Artigo publicado em 31.12.09. 